\title{
Keynotes
}

\section{Selective serotonin re-uptake inhibitors: use in depression}

\author{
I. N. FERRIER, Professor, Department of Psychiatry, School of Neuroscience, University \\ of Newcastle upon Tyne NE1 4LP; T. SILVERSTONE, Professor, Department of \\ Psychological Medicine, St Bartholomew's Hospital, London EC1A 7BE; and \\ D. ECCleston, Professor, Department of Psychiatry, School of Neuroscience, \\ University of Newcastle upon Tyne NE1 4LP
}

This short review outlines the clinical profile of the selective serotonin re-uptake inhibitors (SSRIs). There has been much recent publicity and promotion of this group of drugs and this review attempts to give a balanced account of their current place in the treatment of depression. Although a large number of preclinical and clinical trials have been carried out many questions and problems remain - it is important to proceed carefully and carry out (and replicate) controlled independent clinical trials. The views of general psychiatrists and GPs about these drugs in normal clinical practice will be the acid test - this will be particularly important in view of their cost.

Clomipramine (a tricyclic antidepressant) appeared to be one of the more selective inhibitors of SHT re-uptake but it emerged that its major metabolite exhibited potent neuronal noradrenaline reuptake inhibition. Zimelidine was the first selective 5HT reuptake inhibitor and was shown to have antidepressant activity before it was withdrawn from the market in 1982 on toxicity grounds. There are now five highly selective 5HT uptake inhibitors that have been subjected to intensive clinical investigationcitalopram, fluoxetine, fluvoxamine, paroxetine and sertraline. In general their metabolites are either inactive, do not cross the blood brain barrier or are formed at very low concentration. It seems likely that their clinical effects are due to 5HT re-uptake inhibition although effects on other transmitters cannot be excluded.

There have been numerous studies which indicate that SSRIs are effective antidepressants. The majority have indicated their superiority to placebo and most suggest that the clinical efficacy of this group of drugs is equivalent to that of standard antidepressants, e.g. amitriptyline and imipramine (Aberg-Wistedt, 1989). At present there is no evidence for any differences in clinical efficacy between the SSRIs. Initial reports indicated that SSRIs may have a more rapid action than tricyclic antidepress- ants but the vast majority of studies have not confirmed this. A major issue emerging in the treatment of depression is the question of continuation therapy and prophylaxis and the related question of 'maintenance' doses. As far as SSRIs are concerned, it is still not clear how effective they will prove to be in maintenance therapy-completed studies of large numbers of patients are few - but results so far are promising. Fluoxetine was found to be as effective as imipramine in an open extension of a double-blind trial for up to five years (Wernicke \& Bremner, 1986). Doogan \& Caillard (1988) have shown efficacy for setraline in preventing relapse and recurrence over one year.

The side effects of the SSRIs are similar - the most frequently reported are nausea and vomiting, insomnia, anxiety, sweating, headaches and, surprisingly (in view of their lack of anticholinergic properties), dry mouth. The gastrointestinal side-effects are reported by about $35 \%$ of patients (compared with $10 \%$ of placebo treated patients) but are generally mild, transient and dose-related and may be avoided by gradual increase in dosage. However, some studies report significant problems with nausea leading to non-compliance at higher doses. There are few cardiac or anticholinergic side effects and sedation and hypotension are rare. In view of this profile of side effects, SSRIs are generally well tolerated in the elderly and can be recommended in elderly patients where anticholinergic side-effects and/or hypotension with tricyclics have been or could be a major problem. Weight gain does not occur - indeed weight loss is seen in some studies. Anorgasmia or delayed ejaculation has been reported in a small but significant percentage of treated patients. The sexual problems, which affect both males and females, may be under-reported and this problem merits closer scrutiny. The serious adverse reaction of a 'flu-like drug reaction and Guillain-Barré syndrome which led to the withdrawal of zimelidine has not been seen 
with any of the other SSRIs and while their use is only now becoming widespread there appears to be enough long-term experience with them to indicate that this problem is not shared by the current SSRIs. However a rash, usually urticarial, is seen in a small percentage of patients which, in rare instances, can be associated with fever, arthralgia and lymphadenopathy. If rash occurs the SSRI should be discontinued as more serious systemic events can occur with prolonged use. The rash and any other systemic features usually improve promptly. The dosage of the first SSRIs (fluoxetine and fluvoxamine) was set too high initially and it will be of interest to see if this experience leads to less marked side-effect profiles with the newer SSRIs.

Interactions with most drugs are insignificant but there is an important interaction with lithium which appears to be less problematical with the newer SSRIs (of shorter half-life, e.g. paroxetine and sertraline). The most serious interaction seen with this group of drugs is with the monoamine oxidase inhibitors (MAOIs) where the potentially fatal serotonergic syndrome (characterised by hyperthermia, tremor, myoclonic jerks and finally convulsions) may occur: if a MAOI is a future option it is best to choose an SSRI with a relatively short half-life as this will minimise the washout period before the switch is made.

There was recent concern about the possibility of increased risk of suicide following the administration of one of this group of drugs. The epidemiological data for this is lacking and recently the Food and Drug Administration and the Committee on the Safety of Medicines concluded that there was no evidence of such a risk. There are, in fact, some reports that these drugs reduce suicidal thoughts better than some conventional antidepressants (Montgomery \& Fineberg, 1989) and the SSRIs are much safer in overdose than most conventional tricyclics. Another concern is that these drugs may induce agitation and akathisia, which can be particularly distressing (Lipinski et al, 1989). The frequency of and risk factors for these side-effects, which occur early in treatment, require careful post-marketing surveillance.

A key question about the SSRIs is which patients respond well to them and which do not. This is important for two reasons. First, their place in the therapeutic armamentarium can be defined. Second, this information would be of value in our attempts to elucidate the neurochemistry of major depression. It is clear that while these drugs may be relatively selective in terms of their effects on the 5HT system, they are not clinically specific. Patients with anxiety, panic disorder, obsessive compulsive disorder (OCD) and some eating disorders respond to SSRIs although there are a number of confounding variables in such studies, notably the level of any co-existing depression (Montgomery \& Fineberg, 1989). There is increasing evidence that the lack of clinical specificity is shared by tricyclic antidepressants (particularly those with predominant serotonergic effects which are specifically effective in OCD). Since the SSRIs are associated with an increase in anxiety in some patients there was concern that depressed patients with anxiety and/or agitation may respond unfavourably. The reverse appears to be true and there is evidence that SSRIs are more effective than tricyclics in treating depressed patients with co-existing anxiety (Montgomery \& Fineberg, 1989). Most of the treatment studies on SSRIs report on patients with major depression by DSM criteria with moderate depression rating scores and most studies have been done on out-patients. The question then is whether these drugs are effective in severe depression and/ or psychotic depression and in those who have responded poorly to conventional tricyclics, had frequent relapses or developed a chronic course. These questions remain unanswered in clinical practice and their efficacy in such patients has not been adequately tested as most studies exclude them (as is also the case for tricyclics). There is some evidence that SSRIs are less effective in 'melancholic' type depression (Danish University Antidepressant Group, 1986, 1990) but more recently this has been disputed (Dunbar, 1991; Ottevanger, 1991). One study showed that zimelidine was effective in patients with relapses and previous poor response to other therapies (Hirmatsu et al, 1983) and there are similar reports for fluoxetine and paroxetine (Reimherr et al, 1984; Tyrer et al, 1987) but many more studies are needed. One problem with SSRIs is that these drugs may be less effective than the tricyclics in relieving sleep disturbance in depressed patients, perhaps necessitating the use of hypnotics which may be inappropriate, particularly in the elderly. There is at present no evidence that response to SSRIs can be predicted by using 'biological markers', e.g. CSF 5HIAA.

The present position therefore is that the SSRIs are effective antidepressants with few side-effects and compliance with them is good. They are effective in patients with moderate depression and in patients with mixtures of depression and anxiety. The increase in compliance with SSRIs should allow further examination of the question of whether they are useful for severe depression (in its many forms) and for prophylaxis. In particular it will be of interest to see whether the long-term morbidity of depression can be attenuated by their usage. If this can be established then the considerable increase in cost of these drugs over conventional tricyclics could be justified. The fact that the SSRIs are only as good as tricyclics suggests that the alterations to the 5HT system induced by these drugs are important but not sufficient to produce optimal treatment of depression. 
The current place of these drugs in the treatment of depression has not been established. However it seems that the SSRIs are of established efficacy in moderate depression and are indicated where sideeffects of tricyclics are likely to be a major problem or lead to non-compliance and perhaps where the risk of overdose is high. Safety of drugs is a most important consideration when choosing an antidepressant but efficacy is also an important safety issue as lack of efficacy can lead to episodes of deliberate self-harm. Resolution of this question as well as a comparative cost/benefit analysis will determine the place of these drugs in clinical practice.

\section{References}

ABERG-Wistedt, A. (1989) The antidepressant effects of 5-HT uptake inhibitors. British Journal of Psychiatry, 155, (Suppl 8), 32-40.

DANISH University ANTIDEPRESSANT Group (1986) Citalopram: clinical effect profile in comparison with clomipramine. A controlled multicenter study. Psychopharmacology, 90, 131-138.

- (1990) Paroxetine: a selective serotonin reuptake inhibitor showing better tolerance, but weaker antidepressant effect than clomipramine in a controlled multicenter study. Journal of Affective Disorders, 18, 289-299.

Doogan, D. P. \& Calllard, V. (1988) Sertraline: a new antidepressant. Journal of Clinical Psychiatry, 49 (suppl), 84-87.

Dunbar, G. C. (1991) Paroxetine in the treatment of severe (melancholic) depression. Biological Psychiatry, 29, $255 \mathrm{~S}$.
Hirmatsu, K.-I., Takakashi, R., Mori, A. el al (1983) A multicentre double-blind comparative trial of zimelidine and imipramine in primary major depressive illness. Acta Psychiatrica Scandinavica, 68 (Suppl 308), 41-54.

Lipinski, J., Mallya, G., Zimmerman, P. \& Pope, H. (1989) Fluoxetine-induced akathisia: clinical and theoretical implications. Journal of Clinical Psychiatry, 50, 339-342.

MONTGOMERY, S. A. \& FinEBerg, N. (1989) Is there a relationship between serotonin receptor subtypes and selectivity of response in specific psychiatric illnesses? British Journal of Psychiatry, 155, (Suppl 8), 63-70.

OTteVANGer, E. A. (1991) The efficacy of fluvoxamine in patients with severe depression. British Journal of Clinical Research, 2, 125-132.

Reimherr, F. W., Wood, D. R., Byerley, B., Brainard, J. \& GROsSER, B. I. (1984) Characteristics of responders to fluoxetine. Psychopharmacology Bulletin, 20, 70-72.

Tyrer, P., Marsden, C. A., Casey, P. et al (1987) Clinical efficacy of paroxetine in resistant depression. Journal of Psychopharmacology, 1, 251-7.

WERNICKE, J. F. \& BREMNER, J. D. (1986) Fluoxetine - important in the long term treatment of depression. British Journal of Clinical Practice, 7, 17-23.

A full list of references available on request to Professor Ferrier.

This paper was prepared at the request of the Psychopharmacology Working Party of the Royal College of Psychiatrists and has been approved by the Working Party.

The quotations from the archives which appear throughout this issue of the Psychiatric Bulletin were researched by Susan Floate, Librarian, and Margaret Harcourt Williams, Archivist, RCPsych.

"Resolved that a circular be sent to the Members informing them that in future no annual subscription will be required from them". (The subscription was reestablished, at 1 guinea per member, at the adjourned meeting in 1852.) (1843, annual meeting).

"Present (only) Dr Diamond, Metropolitan Secretary". (He adjourned the meeting to Oxford ten days later, where it was well attended.) (1852, annual meeting).
"We know this, that women have much less pay than men, but what is small pay to a man would be very large pay to a woman. I know several asylums which have been infinitely improved by the introduction of women". (G. W. Mould of Manchester Royal Asylum on women attendents, 1876).

"With regard to inducements, I try different methods. I don't think myself there is a great deal in high pay, but there is I think in the pensions". (Dr Parsey of Hatton, Warwickshire, on Asylum Attendents, 1876). 